\section{Concussions and suicide}

Fralick and colleagues ${ }^{1}$ report a suicide rate three times the population norm for concussions that occur on weekdays and four times the norm for those that occur on weekends. The conclusion was based on a 20-year longitudinal study that used vital statistics from Canada's Office of the Registrar General for the general population and physician claims for ICD-9 code 850, for concussion, for the cohort.

The authors excluded patients under 17 years of age "because most suicide deaths occur in adults." Was a similar exclusion applied to the general population? If not, the exclusion of those less likely for suicide would seem to bias the cohort for a higher rate of suicide.

In stating "This code [850] has been validated with high specificity (99\%) and mid-range sensitivity (22\%-76\%)," the authors cite three references. In one, the authors conclude, "The identification of mild TBI [traumatic brain injury] patients using retrospectively assigned ICD-9 codes appears to be inaccurate. These codes are associated with a significant number of false-positive and falsenegative code assignments."

In another, the authors conclude, "ICD-9-CM codes were sensitive for the presence of any severe TBI, but further classification of specific types of TBI was limited by variable sensitivity/specificity. Use of these codes should be supplemented by other methodology." 3

These cited observations do not seem to promote comfort with Fralick and colleagues' use of retrospective ICD-9 code (850) for defining the cohort.

Nor should this surprise many who have experience with acute closed head trauma. Using the 850 code puts into the same category patients who complain of feeling "out of it" for a few seconds after striking their head while denying a loss of consciousness (850.0) with those who have documented unresponsiveness for more than an hour with no memory of the preceding events (850.4). This indiscriminate comingling of disparate presentations invites skepticism of the predictive power of ICD-9 code 850 . The authors appear to attempt to mitigate the broadness of the concussion diagnosis by excluding patients admitted immediately or within two days after initial assessment, thereby creating an ambulatory subset of ICD-9 code 850 .

The article by Fralick and colleagues invites consideration of the NIOSH (National Institute for Occupational Safety and Health) report by Baron and associates. ${ }^{4}$ The cohort for this study was 3439 former National Football League (NFL) players with five years or more credited playing seasons between 1959 and 1988. The mortality for this cohort was compared with the general mortality for the US population. No consideration for a history of concussion was made in this study. The authors report that the number of suicides occurred at a substantially lower rate (standardized mortality ratio 0.41 ) than that in the general population. If this study is considered in light of the Fralick report of a suicide rate for those who have had a concussion three times higher than that in the general population, one must infer that those who have played five or more years of NFL have experienced concussions at a rate onesixth that of the general US population.

\section{Francis X. Speidel MD}

Drexel University, Philadelphia, Penn.

\section{References}

1. Fralick M, Thiruchelvam D, Tien HC, et al. Risk of suicide after a concussion. CMAJ 2016;188:497-504.

2. Bazarian JJ, Veazie P, Mookerjee S, et al. Accuracy of mild traumatic brain injury case ascertainment using ICD-9 codes. Acad Emerg Med 2006;13:31-8.3

3. Carroll CP, Cochran JA, Guse CE, et al. Are we underestimating the burden of traumatic brain injury? Surveillance of severe traumatic brain injury using Centers for Disease Control International Classification of Disease, ninth revision, clinical modification, traumatic brain injury codes. Neurosurgery 2012;71:1064-70.

4. Baron SL, Hein MJ, Lehman E, et al. Body mass index, playing position, race, and the cardiovascular mortality of retired professional football players. Am J Cardiol 2012;109:889-96.

CMAJ 2016. DOI:10.1503/cmaj.1150101

\section{The authors respond}

Speidel raises some skeptical points about our methods ${ }^{1}$ because we lack clinical details for the 235110 patients included in our study.
We agree that more research is justified because concussions can vary in symptoms, severity, context, construal and recovery. We also agree that ascertainment of suicide is challenging, reflects an uncertain number of falsenegative results and causes most studies to underestimate the risk of suicide.

Speidel invites us to consider the article by Baron and associates. ${ }^{2}$ We agree that this study is widely misquoted and appreciate the opportunity to correct several common misunderstandings. First, the study examined cardiovascular mortality and was not powered to investigate self-harm events. Second, the article does not discuss suicide and relegates intentional self-harm to a single line in one table. Third, the overall mortality from all other causes of violent death was distinctly low, as would be expected among wealthy, retired professional athletes. Fourth, the study ended follow-up in 2007 and excluded at least nine former NFL players who committed suicide in the past five years alone (Jovan Belcher, Kurt Crain, Mike Current, Dave Duerson, Ray Easterling, Paul Oliver, Lawrence Phillips, Adrian Robinson and Junior Seau).

The statement from Speidel has one more caveat, since almost half of those included by Baron and associates were African-American men, a group historically that had a low suicide rate during the study enrolment interval (19591988). That may explain why the authors never stated that NFL players "experienced concussions at a rate one-sixth that of the general US population." Otherwise, the authors might imply excess suicide deaths in their sample.

\section{Donald A. Redelmeier MD \\ Homer C. Tien MD \\ Michael Fralick MD}

Evaluative Clinical Sciences, Sunnybrook Research Institute, Toronto, Ont.

\section{References}

1. Speidel FX. Concussions and suicide [letter CMAJ 2016;188:605.

2. Baron SL, Hein MJ, Lehman E, et al, Body mass index, playing position, race, and the cardiovascular mortality of retired professional football players. Am J Cardiol 2012;109:889-96.

CMAJ 2016. DOI:10.1503/cmaj.1150102 